\title{
Bladder perforation after immediate postoperative intravesical instillation of mitomycin C
}

\author{
Darwin Lim, MD; ${ }^{*}$ Jonathan I. Izawa, MD, FRCSC;, Paul Middlebrook, MD, FRCSC,; \\ Joseph L. Chin, MD, FRCSC*
}

\begin{abstract}
Intravesical chemotherapy after transurethral resection of a bladder tumour (TURBT) has been observed to significantly decrease recurrence rates compared to TURBT alone. Though immediate postoperative intravesical treatment with chemotherapeutic agents after transurethral resection for superficial bladder carcinoma is generally considered a safe and effective adjunctive therapy in decreasing recurrence rates, its instillation is not always completely innocuous. Lately, a more serious complication of bladder perforation associated with immediate instillation of intravesical mitomycin C (MMC) after TURBT was reported. We report our own experience of a male patient with bladder perforation after an early instillation of a single dose of MMC. In this case, systemic toxicity occurred which required intensive care after surgical repair.
\end{abstract}

Can Urol Assoc J 2010;4(1):E1-E3

\section{Introduction}

The recurrence rate of $\mathrm{Ta} / \mathrm{T} 1$ transitional cell cancer after transurethral resection of a bladder tumour (TURBT) alone is around $50 \%$ to $70 \%$ within 5 years postoperatively, with up to $20 \%$ of cases showing pathologically muscle invasive disease.

Since tumour cell implantation during or after transurethral resection (TUR) is considered an important factor for tumour recurrence, immediate postoperative intravesical treatment with chemotherapeutic agents after TUR for superficial bladder carcinoma is considered the standard of care. The procedure is safe and effective in decreasing recurrence rate, however, this is not always completely innocuous. ${ }^{1-3}$ We describe a case wherein the patient experienced a serious complication after immediate postoperative instillation of mitomycin $\mathrm{C}$ (MMC).

\section{Case report}

A 79-year-old man had a history of recurrent TaG2 bladder cancer successfully managed by TURBT (a total of 8 prior procedures) and immediate postoperative instillation of intravesical MMC. The patient was not diabetic and had had no prior cardiac events, but did have peripheral vascular disease with some intermittent claudication. He also suffered from activity-limiting chronic obstructive pulmonary disease and required the use of inhaled steroid and bronchodilators, but no oral steroids. On this occasion, he presented again with a superficial bladder cancer and underwent cold cup biopsy and fulguration of 2 small recurrences of transitional cell carcinoma (stage TaG2). The biopsy site did not reveal perivesical fat. In view of the history of multiple tumour recurrences, the attending surgeon felt that further efforts to minimize the probability of tumour recurrences were indicated, even though the tumours were rather small on this occasion. Immediate postoperative instillation of $40 \mathrm{mg} \mathrm{MMC} \mathrm{in} 40 \mathrm{cc}$ of sterile water was performed in the recovery room 1.5 hours postoperatively. There was no impression of intra-operative perforation. The catheter was removed following 1 hour of MMC instillation.

One day later, the patient complained of severe pelvic and suprapubic pain. A Foley catheter was inserted and cystogram revealed extraperitoneal extravasation. Followup cystogram after 9 days revealed a wide-mouthed diverticulum anteriorly with no evident extravasation. Pain recurred after the catheter was removed. Cystoscopy revealed a non-healed perforation. The patient was recatheterized with plan of urinary drainage for 3 weeks.

However, the patient continued to experience pain, anorexia and developed a fever. He developed leukocytosis while urine culture was positive for Staphylococcus aureus. The appropriate antibiotics were started. As fever persisted, he underwent exploratory laparotomy which revealed retropubic abscess with a large necrotic defect in the anterior bladder wall. The bladder defect was repaired with closesuction drainage for the retropubic area. The retropubic drain began draining urine within 1 week. Urine cultures grew gram negative bacteria and anaerobes. Transurethral placement of 
bilateral 7 French ureteric catheters was performed to achieve effective urinary diversion.

Parenteral nutrition was started with gradual reversal of the patient's catabolic state. The Foley catheter was removed 1 month later. The patient was discharged after a prolonged admission in the rehabilitation unit with ongoing irritative voiding symptoms.

\section{Discussion}

By inducing an inflammatory response in a murine bladder and subsequently instilling tumour cell intravesically, one experimental study demonstrated tumour cell implantation was significantly more susceptible in an inflamed urothelium than normal bladder mucosa. ${ }^{4}$ Soloway and colleagues showed that intravesical chemotherapy significantly decreased the incidence of tumour cell implantation. ${ }^{5}$

Significant decrease in tumour recurrence rate after one immediate postoperative instillation has been reported by several authors. ${ }^{1-3}$ The timing of immediate postoperative intravesical chemotherapy has also been extensively studied. One study showed instillation of intravesical chemotherapy within 6 hours after TURBT, ${ }^{2}$ while another study showed that early treatment within 24 hours was better than delayed treatment for $\mathrm{Ta} / \mathrm{T} 1$ bladder cancer. ${ }^{6}$

Mitomycin C, a 334-kD alkylating agent that inhibits DNA synthesis, is the most widely used intravesical chemotherapeutic agent. It has been found to decrease tumour recurrence rate by $17 \%$ when a single dose is given postoperatively compared to TURBT alone in a non-muscle invasive bladder tumour. ${ }^{7}$ A dose of $20 \mathrm{mg}$ or $40 \mathrm{mg}$ in $40 \mathrm{cc}$ of sterile water is usually given in the recovery room with a dwell time of 1 hour.

Though immediate intravesical therapy of chemotherapeutic agent after TURBT of superficial TCC is generally safe, adverse events have been reported. ${ }^{8-12}$ In MMC, chemical cystitis and skin rashes have been reported, but rarely occurs. Doherty and colleagues reported that more extensive necrosis of the bladder wall and fat necrosis of extravesical tissue occurred when MMC was given within 24 hours of TUR, than the usual muscle necrosis seen after TUR alone. ${ }^{8}$ Bladder perforation associated with immediate instillation of intravesical MMC after TURB has been reported by several authors. ${ }^{9-12}$

It is common practice in TURBT to carry the resection into the detrusor muscle to ensure tumour clearance where ever possible, and to allow accurate pathological staging. However this procedure may result in iatrogenic bladder perforation, especially if deeper resection of the detrusor muscle is performed. Immediate administration of intravesical chemotherapy may thus result in extravasation.

In our patient, bladder perforation was not suspected at the time of the TURB, especially since the depth of the biopsies was relatively shallow and there was no predisposing factor, such as prior steroid use. A possible mechanism is an area of attenuated muscularis propria leading to necrosis after MMC instillation resulting in secondary perforation. The patient's pre-existing peripheral vascular disease and suboptimal tissue oxygenation may have contributed to the poor bladder healing.

\section{Conclusion}

Though immediate instillation of intravesical MMC after TURB is generally considered safe and effective in decreasing tumour recurrence, urologists should be aware of the possible complications, such as extravasation. When bladder perforation is suspected or identified during TUR, immediate postoperative intravesical therapy should be avoided. Early recognition of signs and symptoms of bladder perforation, such as abdominal pain, ileus and signs of peritonitis, is of paramount importance. Vigilant monitoring of patients who receive an immediate postoperative instillation and educating nursing personnel are warranted. Successful management entails prompt catheter reinsertion and consideration of possible ancillary procedures, such as open drainage and bladder repair.

From the *Departments of Surgery \& Oncology, Divisions of Urology \& Surgical Oncology, Schulich School of Medicine \& Dentistry, University of Western Ontario, London, ON; †The Grey-Bruce Health Services, Owen Sound, ON

Competing interests: None declared.

This paper has been peer-reviewed.

\section{References}

1. Oosterlinck W, Kurth KH, Schroder F, et al. A Prospective European Organization for Research and Treatment of Cancer Genitourinary Group Randomized Trial Comparing Transurethral Resection followed by a Single intravesical Instillation of Epirubicin or Water In Single Stage Ta, TI Papillary Carcinoma of the Bladder. J Urol 1993;149:749-52.

2. Solsona E, Iborra I, Ricos JV, et al. Effectiveness of A Single Immediate Mitomycin C Instillation In Patients With Low Risk Superficial Bladder Cancer: Short And Long Term Follow Up. J Urol 1999;161:1120-3.

3. Rajala P, Kaasinen E, Raitanen M, et al. Perioperative Single Dose Instillation of Epirubicin or Inteferon -Alpha after Transurethral Resection for the Prophylaxis of Primary Superficial Bladder Cancer Recurrence: A Prospective Randomized Multicenter Study-FInnBladder III Long Term Results. J Urol 2003;168:981-5.

4. Weldon TE, Soloway MS. Susceptibility Of Urothelium To Neoplastic Cellular Implantation. Urology 1975;5:824-7.

5. Soloway MS, Martino C. Prophylaxis Of Bladder Tumour Implantation. Urology 1976;7:29-34

6. Bouffioux C, Kurth KH, Bono A, et al. Intravesical adjuvant Chemotherapy For Superficial transitional cell Bladder carcinoma: Results Of two European Organization for Research and Treatment of Cancer Genitourinary Group Randomized Trials With Mitomycin C and Doxorubicin comparing Early versus Delayed Instillations And Short term Versus long term Treatment. J Urol 1995;135:934-41.

7. Hall MC, Chang S, Dalbagni G, et al. Guideline For the Management of Non Muscle Invasive Bladder Cancer(Stages Ta, Tl And Tis): 2007 Update. J Urol 2007;178;2314-21. 
Bladder perforation after intravesical instillation of mitomycin C

8. Doherty AP, Trendell-Smith N, Stirling R, et al. Perivesical Fat Necrosis After Adjuvant Intravesical Chemotherapy. BJU Int 1999;83:420-3.

9. Cliff AM, Romaniuk CS, Parr NJ. Perivesical Inflammation After Early Mitomycin C Instillation. BJU Int 2000;83:556-7.

10. Nieuwenhuizzen JA, Bex A, Horenblas S. Unusual Complication After Immediate Postoperative Intravesical Mitomycin C Instillation. Eur Urol 2003;43;711-2.

11. Racioppi M, Porreca A, Foschi N, et al. Bladder Perforation: A Potential Risk Of Early Early Endovesical Chemotherapy with Mitomycin C. Urologia Internationalis 2005;75:373-5.
12. Shapiro O, Jones K, Wang C, et al. Risk of Post-operative Intravesical Mitomycon C Instillation Following Transurethral Bladder resection. Can J Urol 2006;13:3317-20.

Correspondence: Dr. Joseph Chin, Professor, Departments of Surgery \& Oncology, Divisions of Urology \& Surgical Oncology, The University of Western Ontario, London Health Sciences CentreVictoria Hospital, 800 Commissioners Rd. E., Suite (3-120, London, ON N6G 4A5; fax: 519-6858455; joseph.chin@|hsc.on.ca 